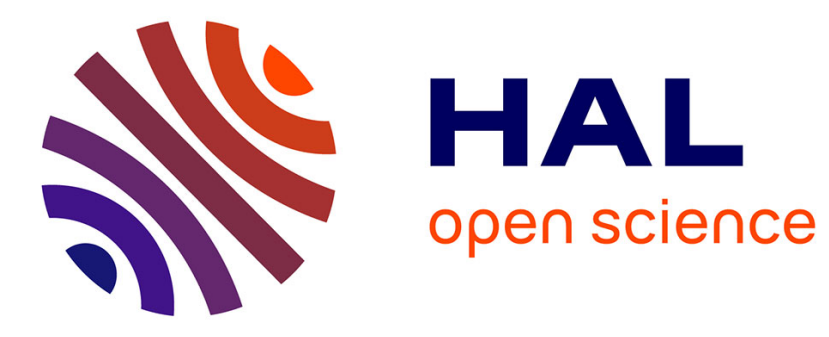

\title{
Displacement Measurement Technique for Beam Kinematics
}

\author{
François Hild, Stéphane Roux, Renaud Gras, Néstor Guerrero, Maria Eugenia \\ Marante, Julio Florez-Lopez
}

\section{- To cite this version:}

François Hild, Stéphane Roux, Renaud Gras, Néstor Guerrero, Maria Eugenia Marante, et al.. Displacement Measurement Technique for Beam Kinematics. Optics and Lasers in Engineering, 2009, 47, pp.495-503. 10.1016/j.optlaseng.2008.03.006 . hal-00322196

\section{HAL Id: hal-00322196 https://hal.science/hal-00322196}

Submitted on 16 Sep 2008

HAL is a multi-disciplinary open access archive for the deposit and dissemination of scientific research documents, whether they are published or not. The documents may come from teaching and research institutions in France or abroad, or from public or private research centers.
L'archive ouverte pluridisciplinaire HAL, est destinée au dépôt et à la diffusion de documents scientifiques de niveau recherche, publiés ou non, émanant des établissements d'enseignement et de recherche français ou étrangers, des laboratoires publics ou privés. 


\title{
Displacement Measurement Technique for Beam Kinematics
}

\author{
François Hild ${ }^{\mathrm{a}, *}$, Stéphane Roux ${ }^{\mathrm{a}}$, Renaud Gras ${ }^{\mathrm{a}, \mathrm{b}}$, \\ Néstor Guerrero $^{\mathrm{b}}$, Maria Eugenia Marante ${ }^{\mathrm{b}}$, \\ Julio Flórez-López ${ }^{\mathrm{c}}$ \\ ${ }^{\mathrm{a}}$ Laboratoire de Mécanique et Technologie (LMT-Cachan) \\ Ecole Normale Supérieure de Cachan / CNRS-UMR 8535 / Université Paris 6 \\ 61 Avenue du Président Wilson, F-94235 Cachan Cedex, France \\ ${ }^{\mathrm{b}}$ Department of Structural Engineering, Lisandro Alvarado University \\ Barquisimeto, Venezuela \\ ${ }^{\mathrm{c}}$ Department of Structural Engineering \\ University of Los Andes Tulio Febres Codero, Mérida 5101, Venezuela
}

\begin{abstract}
It is proposed to develop a digital image correlation procedure that is suitable for beams whose kinematics is described by an Euler-Bernoulli hypothesis. As a direct output, the degrees of freedom corresponding to flexural and axial loads are directly measured. The performance of the correlation algorithm is evaluated by using a picture of a cantilever beam experiment. One load level is analyzed with the present algorithm. The latter is validated by comparing the displacement field with that given by a finite element based correlation algorithm. It is also shown that a locally buckled zone is detectable with the present procedure.
\end{abstract}

Key words: Beam flexure, Digital Image Correlation, generalized degrees of 
freedom, integrated approach, post-processing

\section{Introduction}

The evaluation of damage states of buildings, bridges, off-shore platforms or other civil engineering structures is of paramount importance either in-service or after having experienced an earthquake, since the safety of people is concerned. To perform this task, numerical tools are utilized more and more frequently, yet different routes are followed. The finite element method [1] is one of the classical tools to analyze civil engineering structures. $2 \mathrm{D}[2,3]$ or even 3D $[4,5]$ codes are run. Furthermore, to be realistic, non linear damage models are considered $[6,7]$. From a numerical stand point, different strategies are developed to analyze damage localization and crack propagation, namely, by using eXtended FEM [8] applied to cohesive models [9], embedded models [10] or cohesive segments [11]. Last, the ground motion allows one to evaluate the boundary conditions. Various techniques are used to solve the elastodynamic wave propagation, namely the finite difference method $[12,13]$, the boundary element method $[14,15]$, the finite element method $[16]$ or both $[17,18]$ when the whole interaction between the structure and its foundation is sought.

Simplified or semi-global analyses are another class of methods in which the kinematic fields are described by generalized degrees of freedom (e.g., displacements and rotations in beams or plates). For beams, Euler-Bernoulli or Timoshenko [19] kinematics is classically assumed. The non linear constitutive laws are then integrated over the height of the element. Special, co-called

\footnotetext{
* Corresponding author, Email: francois.hild@lmt.ens-cachan.fr
} 
multi-fiber, beam elements are then implemented [20-23]. Lumped damage mechanics (LDM) is another way to determine the damage state of this type of structures [24-26]. It is based on an extension of the concept of plastic hinges in beam calculations. It is able to account for softening as in other damage theories [27] or due to local buckling [28]. Up to now, the parameters of the constitutive law of hinges, and more generally of beams, relied upon global data such as displacements at few prescribed measurement points [29]. It is proposed to develop and use full-field measurement techniques tailored for the description of these structures as a set of beams and joints.

Digital Image Correlation (DIC) allows one to estimate full displacement fields based on a series of digital images of the surface of a specimen subjected to a specific loading history [30]. Recent advances have been achieved through a novel formulation that enables one to decompose the searched displacement field onto a suited library of such fields [31]. The latter are either finite element shape functions [32], which open the way to a further identification step, or mechanically significant fields suitable for the modeling of frames. The interest of this experimental tool is that it provides full kinematic fields. This type of technique is used to study cracks in brittle materials (e.g., in ceramics [33]) or in brittle structures (e.g., bridges [34]). It is worth noting that enriched kinematics (e.g., à la X-FEM [35]) is also possible. For experiments on beams or frames, it thus gives access to a wealth of data that may be exploited, for example, to identify hinge models at different scales. In the present case, only global degrees of freedom are sought. A first route is given by post-processing measured displacement fields with a standard least squares technique. An alternative route consists in using the relevant displacement basis directly at the measurement stage. Therefore there is no decoupling between the mea- 
surement and identification stages in terms of global degrees of freedom.

In the following, the procedure is illustrated with the analysis of a flexural test on a steel beam. In Section 2, the basic kinematic hypotheses are introduced. They consist of Euler-Bernoulli flexure coupled with tension / compression modes. The measurement procedures used herein are introduced in Section 3. They are based upon a linearized version of the optical flow conservation. Two tools are used, namely a "finite element" procedure and a so-called EulerBernoulli procedure. The first one is generic and is utilized as a validation tool for the second one, which is specialized to beam analyses. The performances in terms of displacement uncertainties are evaluated for both of them. A practical case is analyzed in Section 4. By comparing the results obtained by following the two routes, the new measurement technique is validated and its advantages are discussed.

\section{Beam kinematics}

In the framework of LDM, frames and buildings are modeled as a set of joints, hinges and beams whose kinematics is described by generalized degrees of freedom [19]. In the following, the kinematics of a single beam is addressed. Let us consider a straight bar (i.e., a beam element) of uniform cross section and length $\ell$, which is loaded by axial forces and bending moments about one axis of its cross section. Six degrees of freedom are considered for the bar, namely, two axial displacements, two vertical displacements and two rotations about one axis of the cross section (Figure 1). The local coordinate system $(x, y, z)$ coincides with the principal axes of the cross section, with the $x$-axis representing the centroidal axis of the beam element, and the $z$-axis corresponding 
to the direction of the bending moment.

\subsection{Tension / compression}

Since there are only two nodal displacements $q_{1}$ and $q_{4}$ along the longitudinal direction, a linear variation of the displacement $\tilde{u}$ is assumed with the conditions (Figure 1)

$$
\tilde{u}(x=0)=q_{1} \quad \text { and } \quad \tilde{u}(x=\ell)=q_{4}
$$

so that

$$
\tilde{u}(x)=[\tilde{N}(x)]\{\tilde{q}\} \quad \text { with } \quad \tilde{q}^{t}=\left\{q_{1}, q_{4}\right\} \quad \text { and } \quad[\tilde{N}(x)]=\left[1-\frac{x}{\ell}, \frac{x}{\ell}\right]
$$

and the longitudinal strains read

$$
\tilde{\varepsilon}_{x x}=[\tilde{B}]\{\tilde{q}\} \quad \text { with }[\tilde{B}]=\left[-\frac{1}{\ell}, \frac{1}{\ell}\right]
$$

The axial strains induced by the axial displacements are therefore uniform.

\subsection{In plane flexure}

Let us consider four degrees of freedom $q_{2}, q_{3}, q_{5}$, and $q_{6}$, a cubic displacement model results classically from Euler-Bernoulli kinematics [19] for a cantilever beam, so that the transverse displacement field $\hat{v}$ is such that (Figure 1)

$$
\begin{aligned}
& \hat{v}(x=0)=q_{2} \quad \text { and } \quad \frac{\mathrm{d} \hat{v}}{\mathrm{~d} x}(x=\ell)=q_{3} \\
& \hat{v}(x=\ell)=q_{5} \quad \text { and } \quad \frac{\mathrm{d} \hat{v}}{\mathrm{~d} x}(x=\ell)=q_{6}
\end{aligned}
$$


The vertical displacement field is then expressed as

$$
\hat{v}(x)=[\hat{N}(x)]\{\hat{q}\}
$$

where $\hat{q}^{t}=\left\{q_{2}, q_{3}, q_{5}, q_{6}\right\}$ and $[\hat{N}(x)]=\left[N_{2}(x), N_{3}(x), N_{5}(x), N_{6}(x)\right]$, with

$$
\begin{array}{cll}
N_{2}(x)=\left(2 x^{3}-3 \ell x^{2}+\ell^{3}\right) / \ell^{3} & , & N_{3}(x)=\left(x^{3}-2 \ell x^{2}+\ell^{2} x\right) / \ell^{2} \\
N_{5}(x)=-\left(2 x^{3}-3 \ell x^{2}\right) / \ell^{3} & , & N_{6}(x)=\left(x^{3}-\ell x^{2}\right) / \ell^{2}
\end{array}
$$

and the rotations, with an Euler-Bernoulli kinematics, read

$$
\theta(x)=\frac{\mathrm{d} \hat{v}}{\mathrm{~d} x}(x)=\left[\frac{\mathrm{d} \hat{N}}{\mathrm{~d} x}(x)\right]\{\hat{q}\}
$$

The corresponding horizontal displacement becomes

$$
\hat{u}(x, y)=-y \theta(x)
$$

and the longitudinal strains read

$$
\hat{\varepsilon}_{x x}(x, y)=\frac{\partial u}{\partial x}(x, y)=-y \frac{\mathrm{d} \theta}{\mathrm{d} x}(x)=[\hat{B}(x, y)]\{\hat{q}\}
$$

where $[\hat{B}(x, y)]=\left[B_{2}(x, y), B_{3}(x, y), B_{5}(x, y), B_{6}(x, y)\right]$, with

$$
\begin{aligned}
& B_{2}(x, y)=-\frac{y}{\ell^{3}}(12 x-6 \ell) \quad, \quad B_{3}(x, y)=-\frac{y}{\ell^{2}}(6 x-4 \ell) \\
& B_{5}(x, y)=\frac{y}{\ell^{3}}(12 x-6 \ell) \quad, \quad B_{6}(x, y)=-\frac{y}{\ell^{2}}(6 x-2 \ell)
\end{aligned}
$$

With the chosen kinematics, the axial strain field induced by flexure is a bilinear function of the horizontal and vertical coordinates. 


\subsection{Summary}

From the above mentioned analysis, the displacement field in a beam element reads

$$
\boldsymbol{u}(\boldsymbol{x})=\left[\boldsymbol{N}^{e b}(\boldsymbol{x})\right]\{q\}
$$

where $\boldsymbol{N}^{e b}$ are the shape functions associated with the chosen kinematics (i.e., $\left.N_{1}, \ldots N_{6}, B_{2}, \ldots B_{6}\right)$, and $\{q\}^{t}=\left\{q_{1}, \ldots q_{6}\right\}$. The rotation field is only caused by flexure [Equation (7)]. Last, the longitudinal strain field is expressed as

$$
\varepsilon_{x x}(x, y)=\left[B_{e b}(x, y)\right]\{q\}
$$

where $B_{e b}=\left\{B_{1}, \ldots B_{6}\right\}$. Therefore, the generalized degrees of freedom $\{q\}$ enable for expressing the displacement, rotation and strain fields.

\section{Kinematic measurements by using DIC}

Let us consider a reference image, defined as $f$, i.e., a gray level distribution. An in-plane displacement field $\boldsymbol{u}(\boldsymbol{x})$ is defined. The passive advection of the texture $f$ by the displacement field creates a "deformed image," $g$, such that

$$
g(x+u(x))=f(x)+b(x)
$$

where $b(\boldsymbol{x})$ is noise induced by image acquisition. In the following, it is assumed that the noise level, $b$, can be neglected either because of its low amplitude with respect to those of $f$ and $g$, or because of its scale separation with significant components of the displacement field. Equation (13) is the local form of the "optical flow conservation."

The problem to address is the determination of the displacement field $\boldsymbol{u}$ from 
the exclusive knowledge of $f$ and $g$. As such, the problem is ill-posed, unless additional assumptions are made on the regularity of the displacement field so that the information is sufficient to determine $u$ with a reasonable accuracy. Let us introduce the following objective functional $\Phi$ operating on displacement fields $\boldsymbol{v}$

$$
\Phi^{2}(\boldsymbol{v})=\iint[g(\boldsymbol{x}+\boldsymbol{v}(\boldsymbol{x}))-f(\boldsymbol{x})]^{2} \mathrm{~d} \boldsymbol{x}
$$

In the absence of noise, this functional reaches its minimum value, 0 , for $\boldsymbol{v}=\boldsymbol{u}$ [see Equation (13)]. The trial displacement $\boldsymbol{v}$ may be any choice as will be discussed below. To fulfill a smoothness assumption on $\boldsymbol{u}, \boldsymbol{v}$ may be low-pass filtered or chosen in a subspace of "suited" (i.e., slowly varying) functions. Let us first assume that $f$ and $g$ are sufficiently smooth at a small scale, and the displacement small enough in amplitude so that a Taylor expansion of $g$ up to the first order is introduced in Equation (14)

$$
\Phi_{\operatorname{lin}}^{2}(\boldsymbol{v})=\iint[g(\boldsymbol{x})-f(\boldsymbol{x})+\boldsymbol{v}(\boldsymbol{x}) \cdot \boldsymbol{\nabla} g(\boldsymbol{x})]^{2} \mathrm{~d} \boldsymbol{x}
$$

Equation (15) corresponds to the linearized objective functional associated with the optical flow conservation. The displacement can only be measured along the direction of the intensity gradient. Consequently, additional hypotheses have to be proposed to solve the problem. A strategy consists in decomposing the displacement field as a linear combination of (chosen) basis functions $\boldsymbol{\eta}_{i}(\boldsymbol{x})$

$$
\boldsymbol{v}(\boldsymbol{x})=v_{i} \boldsymbol{\eta}_{i}(\boldsymbol{x})
$$

where Einstein's convention is used, so that $\Phi_{\text {lin }}^{2}$ becomes a quadratic form in the amplitudes $v_{i}$. The extremality condition thus dictates, for all $j$,

$$
\left[\iint\left(\boldsymbol{\nabla} g \cdot \boldsymbol{\eta}_{j}\right)(\boldsymbol{x})\left(\boldsymbol{\nabla} g \cdot \boldsymbol{\eta}_{k}\right)(\boldsymbol{x}) \mathrm{d} \boldsymbol{x}\right] v_{k}=\iint(f-g)(\boldsymbol{x})\left(\boldsymbol{\nabla} g \cdot \boldsymbol{\eta}_{j}\right)(\boldsymbol{x}) \mathrm{d} \boldsymbol{x}
$$


This system is written in a matrix form as

$$
[M]\{\check{v}\}=\{m\}
$$

where $\{\check{v}\}$ is a vector containing all the unknown components $v_{i},[M]$ and $\{m\}$ are known quantities dependent upon $f, g$, and $\boldsymbol{\eta}$. The use of a Taylor expansion (15) requires that the displacement be small when compared with the correlation length of the texture. For a fine texture and a large initial displacement, this requirement appears as inappropriate to converge to a meaningful solution. Thus one may devise a generalization to arbitrarily expand the correlation length of the texture. This is achieved through a coarse-graining step. Many ways may be considered, such as a low pass filtering in Fourier or Wavelet spaces. A rather crude, but efficient way, is to resort to a simple coarse-graining in real space $[36,32]$ obtained by forming super-pixels of size $2 n \times 2 n$ pixels, by averaging the gray levels of the pixels contained in each super-pixel. The procedure is then applied first to the coarsest picture and then more and more details are added.

\section{1 "Finite Element" displacements: Q4-DIC}

The general method presented in equations (17)-(19) can be applied to a large variety of test functions. Q4-elements defined on a square grid (bi-linear functions of $x$ and $y$ ) are chosen here [32] for comparison purposes. The number of pixels in each element is $\lambda \times \lambda$. The displacement decomposition (16) is particularized to account for the shape functions of a finite element discretization. The basis functions $\boldsymbol{\eta}_{i}(\boldsymbol{x})$ are decomposed into components $N_{i j}^{e}(\boldsymbol{x})$ associated 
with different directions $\boldsymbol{e}_{j}$

$$
\boldsymbol{\eta}_{i}(\boldsymbol{x})=N_{i j}^{e}(\boldsymbol{x}) \boldsymbol{e}_{j}
$$

Generally, the shape functions are such that $N_{i j}^{e}(\boldsymbol{x})=N^{e}(\boldsymbol{x}) \delta_{i j}$, where $\delta$ is the Kronecker delta and $N^{e}$ is the scalar shape function, here given for bilinear elements

$$
N^{e}(x, y)=\frac{1}{4}\left(1 \pm \frac{2 x}{\lambda}\right)\left(1 \pm \frac{2 y}{\lambda}\right)
$$

when $-\lambda / 2 \leq x \leq \lambda / 2,-\lambda / 2 \leq y \leq \lambda / 2$, and $e=1,2,3,4$. The sought amplitudes $v_{i}$ are thus the different components of all the nodal displacements. In the present case, the only parameter the user has to set is the element size $\lambda$. It is worth noting that Equation (15) allows one to evaluate the overall quality of the measurement, as well as local deviations since the quantity is evaluated for each considered pixel. The latter may occur when plastic hinges or cracks appear during the experiment. An example will be discussed in the sequel. When the degrees of freedom $q_{1}, \ldots q_{6}$ are sought, the measured (Q4P1) displacement has to be post-processed.

\subsection{Euler-Bernoulli Kinematics: Beam-DIC}

The results derived in Section 2 allow us to propose a direct approach to the measurement of the degrees of freedom $q_{1}, \ldots q_{6}$ for each beam element. By choosing a priori a basis of functions $\boldsymbol{\eta}_{i}(\boldsymbol{x})$ relevant to an experiment, the identification of the unknown amplitudes $v_{i}$ not only provides an information on the displacement features but also yields quantities to be used for mechanical modeling, say LDM. In the present case, the previous example is analyzed by using an integrated approach. Since the basis function is richer, the number of elements is reduced (it is as low as one) and its size is larger (in the present 
case up to $\ell=2048$ pixels). The chosen basis consists of the displacement field induced by axial and flexural loadings [see Equation (11)]. Consequently, the six unknown degrees of freedom of each beam element are directly identified, instead of evaluated by post-processing the measured displacements. A global quality factor is obtained, and even a local map of non-resolved differences [Equation (15)]. This feature is in turn utilized to identify the best degrees of freedom in the sense of lowest global error $\Phi_{\text {lin }}$.

In practice, a region of interest $(\mathrm{ROI})$ is chosen by the user. The ROI is then subdivided into smaller elements, referred to as zones of interest (ZOIs). The proposed approach is based upon a first order Taylor expansion (15). When the (vertical) displacements are large, it will not be robust. Therefore, a first evaluation of the vertical displacement field is obtained by performing a classical correlation analysis in which, for each ZOI, a uniform displacement is sought. An FFT algorithm is used and a sub-pixel value is determined by interpolating the correlation function in the vicinity of its maximum [36]. At least four ZOIs are used so that the displacement field is interpolated by a cubic polynomial, or equivalently by using the decomposition (5). Each pixel of the ROI is then moved by the estimated displacement. The subsequent displacement residuals are small so that the first order decomposition (5) is robust enough. The linear system (18) is implemented and solved. An iterative scheme is possible for which the displacement of each row of pixels is updated. Convergence is obtained when the global error estimator reaches a stationary value. 


\subsection{Analyzed pictures}

Figure 2a shows a reference picture of a cantilever beam loaded at its end section by an actuator. The beam has a length of $1.28 \mathrm{~m}$, a square cross-section of outer size $120 \mathrm{~mm}$ and inner size $112 \mathrm{~mm}$. It is made of a conventional construction steel (ASTM-A-500). The picture was shot with a digital CMOS camera (resolution: $3888 \times 2592$ pixels, digitization: 8 bits). Figure $2 \mathrm{~b}$ corresponds to a deformed shape of the considered beam. A random texture was applied onto the observed surface of the beam prior to the experiment to improve the measurement uncertainty by creating local gray level fluctuations. The latter are the key to extract displacement fields from pictures.

\subsection{Baseline study for Q4-DIC}

The performance of the correlation algorithm is evaluated by recording a picture prior to the experiment (Figure 2a) and applying artificial (i.e., known) displacements. The considered ROI has a size of $256 \times 2048$ pixels. The displacement uncertainty is estimated when the correlation parameters are modified. A constant displacement varying between 0 and 1 pixel, with an increment of 0.1 pixel is applied artificially by using the shift / modulation property of Fourier transforms. In the present study, the reference picture of Figure $2 \mathrm{a}$ is considered. The displacement uncertainty $\sigma_{u}$, defined as the mean of the standard displacement uncertainties, is plotted as a function of the element

size in Figure 3. A power law with an exponent $\alpha$ of the order 2 is obtained for the displacement uncertainty $\sigma_{u}$ up to an element size $\lambda=32$ pixels

$$
\sigma_{u}=\frac{A^{\alpha+1}}{\lambda^{\alpha}}
$$


with $A=1$ pixel, thereby indicating that the displacement uncertainty $\sigma_{u}$ and the spatial resolution $\lambda$ are the result of a compromise. By using the displacement interpolation (20), the average strain in one element is easily computed. The standard strain uncertainty $\sigma_{\varepsilon}$ is then expressed as

$$
\sigma_{\varepsilon}=B \frac{\sigma_{u}}{\lambda}
$$

where the constant $B$ is of the order of $\sqrt{2}$ [37]. A lower bound is obtained by using the results of Equation (21). When an element size of 16 pixels is chosen, the standard strain uncertainty is of the order of $3.5 \times 10^{-4}$. Therefore, local strain levels less than $3 \sigma_{\varepsilon} \approx 10^{-3}$ cannot be measured accurately with the chosen element size.

\subsection{Baseline study for Beam-DIC}

The same type of analysis is performed with the integrated algorithm. There are however some differences. In the present case, the element height is fixed (here equal to 256 pixels, i.e., less than the height of the beam) and the number of elements along the axial direction is changed. The length of the elements is denoted by $\ell$. Since there are more degrees of freedom related to transverse displacements, namely 4 out of 6 unknowns, constant transverse displacements varying between -0.5 and 0.5 pixel, with an increment of 0.1 pixel is applied. The displacement uncertainty is measured on each component of the displacement field. The maximum value between the two directions is recorded and plotted as a function of the element length in Figure 4. A power law with an exponent of 0.6 is obtained for element lengths greater than or equal to 16 pixels. Since only one direction is affected by the size variation, the decrease is not as steep as in the previous case (Figure 3). However, the overall level 
is comparable to that observed with a Q4 interpolation, and reaches similar values for large element sizes. For smaller lengths ( $\leq 8$ pixels), a divergence occurs, indicating that the chosen basis is too rich to capture accurately a uniform displacement. Globally, prescribing a genuine kinematics allows one to reduce significantly the standard uncertainty for moderate element sizes.

\section{Analysis of an experiment}

In the following, the two pictures of Figure 2 are analyzed. The ROI is centered along the centroid direction and occupies the left part of the picture. Its size is equal to $256 \times 2048$ pixels. First, the Q4-algorithm is used. Having been tested on several situations $[31,38,32]$, it is believed that it is sufficiently reliable in the present situation. Second, the direct approach will be tested. Being new, it will be compared with the Q4 technique for validation purposes.

\subsection{Q4 approach}

As mentioned above, the only parameter the user has to choose is the element size. In the present case, $\ell=16$ pixels. It is believed to be a good compromise between uncertainty and spatial resolution (Figure 3). The displacement maps

are shown in Figure 5. A dominant flexural loading is observed (Figure 5a), even though some axial loading is applied (Figure 5b). The latter is induced by a coupling that cannot be avoided between tensile / compressive and flexural contributions. For comparison purposes, the degrees of freedom q1 to q6 are needed. They are provided directly by the integrated approach. However, they are to be identified in the present case. The beam kinematics developed in 
Section 2 is used to post-process the measured fields. The difference between measured displacements at points $\boldsymbol{x}_{m e}$ and those determined from the closedform solution (11) is minimized in the least squares sense

$$
\Theta^{2}(\{q\})=\sum_{m e}\left\|\boldsymbol{N}_{i}^{b e}\left(\boldsymbol{x}_{m e}\right) q_{i}-\boldsymbol{u}_{m e}\right\|^{2}
$$

where $\|$.$\| is the classical 2-norm. In the present case, m e=2312$, or 4624 degrees of freedom to be compared with the 6 degrees of freedom associated with Euler-Bernoulli kinematics. The post-processed displacement maps are shown in Figure 6. A good agreement is observed when compared with the original results. The displacement uncertainties do not allow for a direct evaluation of the strain field (see Section 3.4). However, when the displacements are post-processed, the strain field is a straightforward evaluation [by using Equations (3) and (9)]. The axial strain map is shown in Figure 7. As expected from the analysis of the displacement maps, combined axial and flexural components are observed. An average axial strain of $-2.1 \times 10^{-4}$ is identified. Even though the strain uncertainty does not allow for a direct analysis of the strain field derived from the raw displacement field, the corresponding mean value is found to be equal to $-2 \times 10^{-4}$, in good agreement with that obtained after post-processing the displacement field.

\subsection{Direct approach}

To avoid the previous two-step procedure, the direct approach is used. First, only one beam element is chosen (its size is therefore equal to $256 \times 2048$ pixels). The initial residual map [when $\boldsymbol{v}=\mathbf{0}$ in Equation (14)] is shown in Figure 8a. An average value of 28 gray levels is obtained. At the end of the calculation that required two iterations of the direct algorithm, the corresponding average 
is less than 7 gray levels. The map is shown in Figure 8b. It is worth noting that its distribution is spatially uniform. The results are deemed trustworthy according to the chosen criterion. The displacement maps are shown in Figure 9. They are very similar to those directly measured by using the Q4algorithm (Figure 5) or post-processed (Figure 6). This result constitutes yet another validation of the direct approach. By using Equations (7) and (12), the section rotation and the axial strains are obtained directly by using the six degrees of freedom. Figure 10 shows the corresponding maps. As expected, a small rotation occurs on the left end of the considered ROI. An average axial strain of $-2.2 \times 10^{-4}$ is measured. This value is in good agreement with that found in the previous subsection (i.e., $\left.-2.1 \times 10^{-4}\right)$.

The number of elements is now changed. Figure 11 shows the mean residual as a function of the number of elements. As the number of elements increases, so does the number of degrees of freedom, yet the residuals are virtually constant. From this result, it is concluded that the measurements obtained with one element is accurate and only 6 degrees of freedom are sufficient to characterize the whole displacement field. The simple kinematics chosen herein is thus validated for the analyzed load level.

The effect of the number of elements on the evaluation of the degrees of freedom corresponding to the left and right ends of the ROI is shown in Figure 12. The values of the six degrees of freedom are very close even though the total number of degrees of freedom increases and its evaluation may become less secure. It is not the case herein because the number of pixels in each element is still very large compared to the number of measured degrees of freedom.

When the strains are evaluated, the average value only varies less than $10 \%$ of 
the observed level with one element for the cases considered. Let us underline the stability of these determinations as a function of the chosen bases, even when using Q4 results. However, two regions appear in terms of standard deviation (Figure 13). When more than one element is used, the continuity of the strain field is not prescribed with the chosen beam kinematics (i.e., only translations and rotation are continuous). Therefore, the analysis of the strain fluctuations is an indication of the overall quality of the measurement. For a number of elements less than or equal to 8, the strain fluctuations are very close. Beyond that number, the latter is more affected by the change of the number of elements. It is an indirect consequence of the results shown in Figure 4 .

When average quantities of various maps are analyzed, the effect of the number of elements is not significant (Figure 14). It is less than $10^{-3}$ pixel. The error bars correspond to the fluctuations of the considered field. Let us underline the very good stability of these determinations as functions of the chosen basis. All these results validate the direct approach proposed herein.

\subsection{Detection of locally-buckled zone}

A second case is considered when the applied load level is significantly higher (see Figure 2-b) than in the previous analysis. The same ROI is considered in the present situation. First, the residual error map is analyzed. In Figure 15, the initial map prior to any correlation analysis and at convergence are shown. As expected, the initial error level is very high. At convergence, the average

level is comparable to the case analyzed previously, except in the left part of the ROI (dashed box), in which the average error is twice that in the other 
part. This result constitutes a first warning that the chosen kinematics is not able to capture the actual kinematics in that zone.

Second, the longitudinal displacement and rotation fields are shown in Figure 16. In the left zone (dashed box) deemed inaccurate according to the residual error level (Figure 15-b), the displacement field does not correspond to classical results, namely, one would assume a constant longitudinal displacement along the height of the sample near the weld. This is not the case. Furthermore, the rotation field is also biased since one would expect very small rotation levels close to the weld, and not about one half of that at the other end of the ROI. All these results confirm that the chosen (i.e., Euler-Bernoulli) kinematics is not able to describe the displacement field in the left part of the beam.

The phenomenon occurring in that zone corresponds to local buckling that is not accounted for by the simple kinematics assumed herein. To analyze accurately the kinematics in that zone, one would need to resort to a full 3D evaluation of the displacement field by using, for instance, stereovision $[41,42]$. If the present means are used, the kinematics of the hinge, lumped at the base of the weld, may be evaluated by discarding the incriminated zone in the correlation analysis and then extrapolating the rotation field at the point of interest, namely, the base of the weld. This procedure would then give the information needed to determine the plastic hinge behavior in the pre- and post-buckled regimes for a subsequent use in frame calculations [43]. This work is underway. 


\section{Summary}

The analysis of Euler-Bernoulli displacement fields based on digital image correlation has been described in terms of a post-processing analysis, and a direct approach. In both cases, the definition of a suited library of displacement field reveals to be a key ingredient for an accurate and reliable evaluation of the beam kinematics in terms of generalized degrees of freedom. When applied to a real experiment, the feasibility is demonstrated by using two pictures obtained with a pocket camera. The direct approach was validated thanks to the Q4 algorithm. It was also shown that when one departs from the kinematic hypotheses made herein, the correlation residuals are a good error indicator. In present case, it allows for the detection of local buckling.

The methodology used in this paper is directly applicable to a broad class of different test geometries. This study illustrates the level of accuracy that is obtained on the displacement and strain fields, with a very low cost equipment, and low additional test constraints (simple surface preparation is required, and good lighting condition is sufficient). Let us underline for instance that the maximum axial displacement is less than $0.7 \mathrm{~mm}$, and the corresponding transverse displacement is less than $3.5 \mathrm{~mm}$ for a beam of length $1.28 \mathrm{~m}$ and height $120 \mathrm{~mm}$. The maximum absolute strain level is about $6 \times 10^{-4}$. It was shown that by moving artificially pictures, a displacement uncertainty of the order of $3.5 \mu \mathrm{m}$ is achieved with the chosen parameters.

It would be of interest to extend the analysis to more complex situations to study, for instance, the behavior of hinges. The corresponding kinematics may be implemented, but also more local and detailed analyses are possible 
by using the algorithm presented herein. By considering the kinematics of joints, it would then be possible to analyze the behavior of frames in terms of global and local degrees of freedom. Last, if a distributed damage field is sought, either in terms global parameters or local ones, different identification techniques are available. One of them, the equilibrium gap method [39], allows for the identification of elastic property and damage fields as well as growth laws [40]. A suited adaptation to the chosen scale and kinematics introduced herein is currently in progress.

\section{Acknowledgements}

This work is part of an ECOS-Nord / CNRS project entitled "Detection and prediction of the damage state in frames subjected to earthquakes."

\section{References}

[1] O. C. Zienkievicz and R. L. Taylor, The Finite Element Method, (McGraw-Hill, London (UK), 4th edition, 1989).

[2] H. G. Kwak and F. C. Filippou, Nonlinear FE analysis of R/C structures under monotonic loads, Comput. Struct. 65 [1] (1997) 1-16.

[3] N. Ile, J. M. Reynouard and J. F. Georgin, Non-linear response and modelling of RC walls subjected to seismic loading, ISET J. Earthqu. Technol. 39 [1-2] (2002) 2002.

[4] J. Y. R. Rashid, R. A. Dameron and R. S. Dunham, Finite element analysis of reinforced concrete in bridge seismic design practice, in: Modeling of inelastic 
behavior of RC structures under seismic loads, P. B. Shing and T. Tanabé, eds., (ASCE, 2001), 217-233.

[5] M. Kwon and E. Spacone, Three-dimensional finite element analyses of reinforced concrete columns, Comput. Struct. 80 [2] (2002) 199-212.

[6] J. Mazars and G. Pijaudier-Cabot, Continuum Damage Theory, Application to Concrete, J. Eng. Mech. 115 [2] (1989) 345-365.

[7] F. Légeron, P. Paultre and J. Mazars, Damage Mechanics Modeling of Nonlinear Seismic Behavior of Concrete Structures, J. Struct. Eng. 131 [6] (2005) 946-955.

[8] N. Möes, J. Dolbow and T. Belytschko, A finite element method for crack growth without remeshing, Int. J. Num. Meth. Eng. 46 [1] (1999) 133150.

[9] G. Wells and L. Sluys, A new method for modeling cohesive cracks using finite elements, Int. J. Num. Meth. Eng. 50 (2001) 2667-2682.

[10] M. Jirasek, Comparative study on finite elements with embedded cracks, Comp. Meth. Appl. Mech. Eng. 188 (2000) 307-330.

[11] J. J. C. Remmers, R. de Borst and A. Needleman, A cohesive segments method for the simulation of crack growth, Comput. Mech. 31 (2003) 69-77.

[12] R. Graves, Simulating Seismic wave propagation in 3D elastic media using staggered grid finite differences, Bull. Seism. Soc. Am. 86 (1996) 1091-1106.

[13] K. Olsen and R. Archuleta, Three-Dimensional simulation of earthquakes on the Los Angeles fault system, Bull. Seism. Soc. Am. 86 (1996) 575-586.

[14] H. Kawase, Time-Domain response of a semi-circular canyon for incident SV, P, and Rayleigh waves calculated by the discrete wavenumber boundary element method, Bull. Seism. Soc. Am. 78, (1988) 1415-1437.

[15] H. B. Coda and W. S. Venturini, Three-dimensional transient BEM analysis, Comput. Struct. 56 [5] (1995) 751-768. 
[16] H. Bao, J. Bielak, O. Ghattas, L. Kallivokas, D. O'Hallaron, J. Schewchuk and J. $\mathrm{Xu}$, Large-scale simulation of elastic wave propagation in heterogeneous media on parallel computers, Comp. Meth. Appl. Mech. Eng. 152 (1998) 856102.

[17] H. B. Coda, W. S. Venturini and F. Aliabadi, A General 3D BEM/FEM Coupling Applied to Elastodinamic Continua/Frame Structures Interaction Analysis, Int. J. Num. Meth. Eng. 46 (1999) 695-712.

[18] M. A. Millan and J. Dominguez, Coupled BEM/FEM model for dynamic analysis of large structures founded on piles in viscoelastic or porous saturated soils, Proceedings 17th ASCE Engineering Mechanics Conference, (2004), 8p.

[19] S. P. Timoshenko, Strength of Materials, (Krieger Publishing Company (3rd edition), 1976).

[20] D. R. J. Owen and E. Hinton, Finite elements in plasticity: Theory and practice, (Pineridge Press Ltd, Swansea, UK, 1980).

[21] J. Guedes, P. Pégon and A. Pinto, A fiber Timoshenko beal element in CASTEM 2000, (JRC, I-21020, Ispra, Italy, Special publication No. I.94.31, 1994).

[22] L. Davenne, F. Ragueneau, J. Mazars and A. Ibrahimbegovic, Efficient approaches to finite element analysis in earthquake engineering, Comput. Struct. $81[12]$ (2003) 1223-1239.

[23] P. Kotronis and J. Mazars, Simplified Modelling Strategies to Simulate the Dynamic Behaviour of R/C Walls, J. Earthquake Eng. 9 [2] (2005) 285-306.

[24] A. Cipollina, A. López-Inojosa and J. Flórez-López, A simplified damage mechanics approach to nonlinear analysis of frames, Comput. Struct. 54 [6] (1995) 1113-1126.

[25] M. E. Marante and J. Flórez-López, model of damage for RC elements subjected to biaxial bending, Eng. Struct. 24 [9] (2002) 1141-1152. 
[26] F. Armero and D. Ehrlich, Numerical modeling of softening hinges in thin Euler-Bernoulli beams, Comput. Struct. 84 [10-11] (2006) 641-656.

[27] J. Lemaitre, A Course on Damage Mechanics, (Springer-Verlag, Berlin (Germany), 1992).

[28] I. S. Sohal and W.-F. Chen, Local buckling and sectional behavior of fabricated tubes, ASCE J. Struct. Eng. 113 [3] (1987) 519-533.

[29] M. E. Marante and J. Flórez-López, Three-dimensional analysis of reinforced concrete frames based on lumped damage mechanics, Int. J. Solids Struct. 40 [19] (2003) 5109-5023.

[30] T. C. Chu, W. F. Ranson, M. A. Sutton and W. H. Petters, Applications of Digital-Image-Correlation Techniques to Experimental Mechanics, Exp. Mech. 3 [25] (1985) 232-244.

[31] F. Hild and S. Roux, Digital image correlation: from measurement to identification of elastic properties - A review, Strain 42 (2006) 69-80.

[32] G. Besnard, F. Hild and S. Roux, "Finite-element" displacement fields analysis from digital images: Application to Portevin-Le Châtelier bands, Exp. Mech. 46 (2006) 789-803.

[33] P. Forquin, L. Rota, Y. Charles and F. Hild, A Method to Determine the Toughness Scatter of Brittle Materials, Int. J. Fract. 125 [1] (2004) 171-187.

[34] M. Küntz, M. Jolin, J. Bastien, F. Perez and F. Hild, Digital image correlation analysis of crack behavior in a reinforced concrete beam during a load test, Canad. J. Civil Eng. 33 (2006) 1418-1425.

[35] J. Réthoré, S. Roux and F. Hild, From pictures to extended finite elements: Extended digital image correlation (X-DIC), C. R. Mecanique 335 (2007) 136137. 
[36] F. Hild, B. Raka, M. Baudequin, S. Roux and F. Cantelaube, Multi-Scale Displacement Field Measurements of Compressed Mineral Wool Samples by Digital Image Correlation, Appl. Optics IP 41 [32] (2002) 6815-6828.

[37] S. Bergonnier, F. Hild and S. Roux, Digital image correlation used for mechanical tests on crimped glass wool samples, J. Strain Analysis 40 [2] (2005) 185-197.

[38] S. Roux and F. Hild, Stress intensity factor measurements from digital image correlation: post-processing and integrated approaches, Int. J. Fract. 140 [1-4] (2006) 141-157.

[39] D. Claire, F. Hild and S. Roux, A finite element formulation to identify damage fields: The equilibrium gap method, Int. J. Num. Meth. Engng. 61 [2] (2004) 189-208.

[40] S. Roux and F. Hild, From image analysis to damage constitutive law identification, NDT.net 11 [12] (2006) roux.pdf. See also S. Roux and F. Hild, Digital Image Mechanical Identification (DIMI), to appear in Exp. Mech. (2007).

[41] M. A. Sutton, S. R. McNeill, J. D. Helm and Y. J. Chao, Advances in TwoDimensional and Three-Dimensional Computer Vision, in: Photomechanics, P. K. Rastogi, eds., (Springer, Berlin (Germany), 2000), 323-372.

[42] J.-J. Orteu, T. Cutard, D. Garcia, E. Cailleux and L. Robert, Application of Stereovision to the Mechanical Characterisation of Ceramic Refractories Reinforced with Metallic Fibres, Strain 43 [2] (2007) 96108.

[43] N. Guerrero, M. E. Marante, R. Picón and J. Flórez-López, Model of local buckling in steel hollow structural elements subjected to biaxial bending, $J$. Construct. Steel Res. 63 [6] (2007) 779-790. 


\section{List of Figures}

1 Beam element with 6 degrees of freedom.

2 Cantilever beam in its reference (a) and deformed (b) state.

The picture resolution is $3888 \times 2592$ pixels with an 8 -bit

digitization.

3 Standard displacement uncertainty as a function of element size $\lambda$ using Q4 finite elements. The dashed line corresponds to a power law fit [Equation (21)] with an exponent close to 2 .

4 Standard displacement uncertainty as a function of beam element length $\ell$. The dashed line corresponds to a power law fit with an exponent of the order of 0.6 .

5 Displacement maps in pixels (1 pixel $\equiv 0.35 \mathrm{~mm}$ ) along the longitudinal and transverse directions measured with a Q4-algorithm. Elements of size $\ell=16$ pixels were considered.

6 Post-processed displacement maps using one beam element along the longitudinal and transverse directions. The same color scales as in Figure 5 are chosen.

$7 \quad$ Axial strain map obtained from post-processed displacements using one beam element (Figure 6)

8 Residual error map in gray levels prior to (a) and after (b) performing the correlation. One beam element of length $\ell=2048$ pixels is considered. 
9 Displacement maps along the longitudinal and transverse directions. One beam element of length $\ell=2048$ pixels is considered. The same color scales as in Figure 5 are chosen.

10 Rotation (a) and axial strain (b) maps (the same color scale as in Figure 7 is chosen). One beam element of length $\ell=2048$ pixels is considered.

11 Average residual (in gray levels) as a function of number of beam elements. The average initial residual (i.e., the picture difference) is equal to 28 gray levels.

12 Degrees of freedom as functions of number of beam elements.

13 Average axial strain as a function of number of beam elements. The error bars correspond to the root mean square values.

14 Average displacements as functions of number of beam elements. The error bars correspond to the root mean square values.

15 Residual error maps prior to correlation (left) and after convergence (right) for the deformed picture shown in Figure 2. The dashed box shows the zone where the correlation result is not accurate. One beam element of length $\ell=2048$ pixels is considered. 
16 Displacement map along the longitudinal direction and corresponding rotation map. The dashed box shows the zone where the measured kinematics is not trustworthy. One beam element of length $\ell=2048$ pixels is considered. 


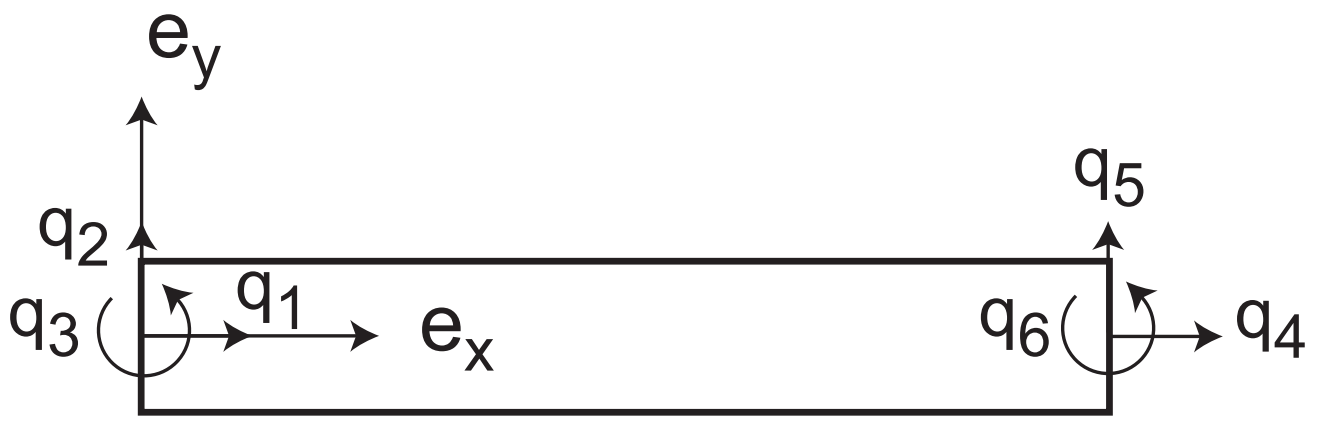

Fig. 1. Beam element with 6 degrees of freedom. 

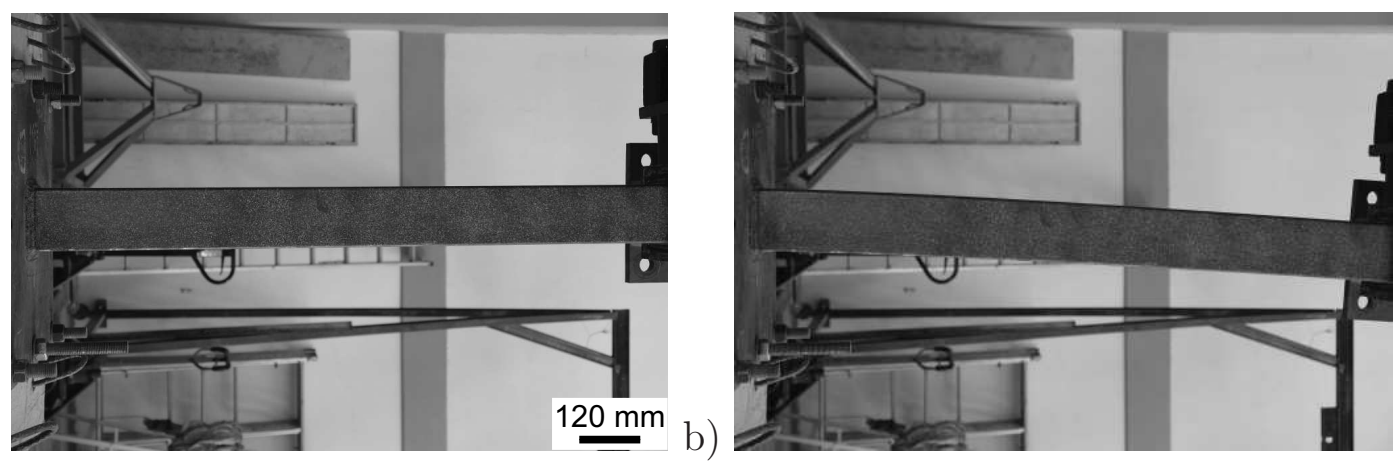

Fig. 2. Cantilever beam in its reference (a) and deformed (b) state. The picture resolution is $3888 \times 2592$ pixels with an 8 -bit digitization. 


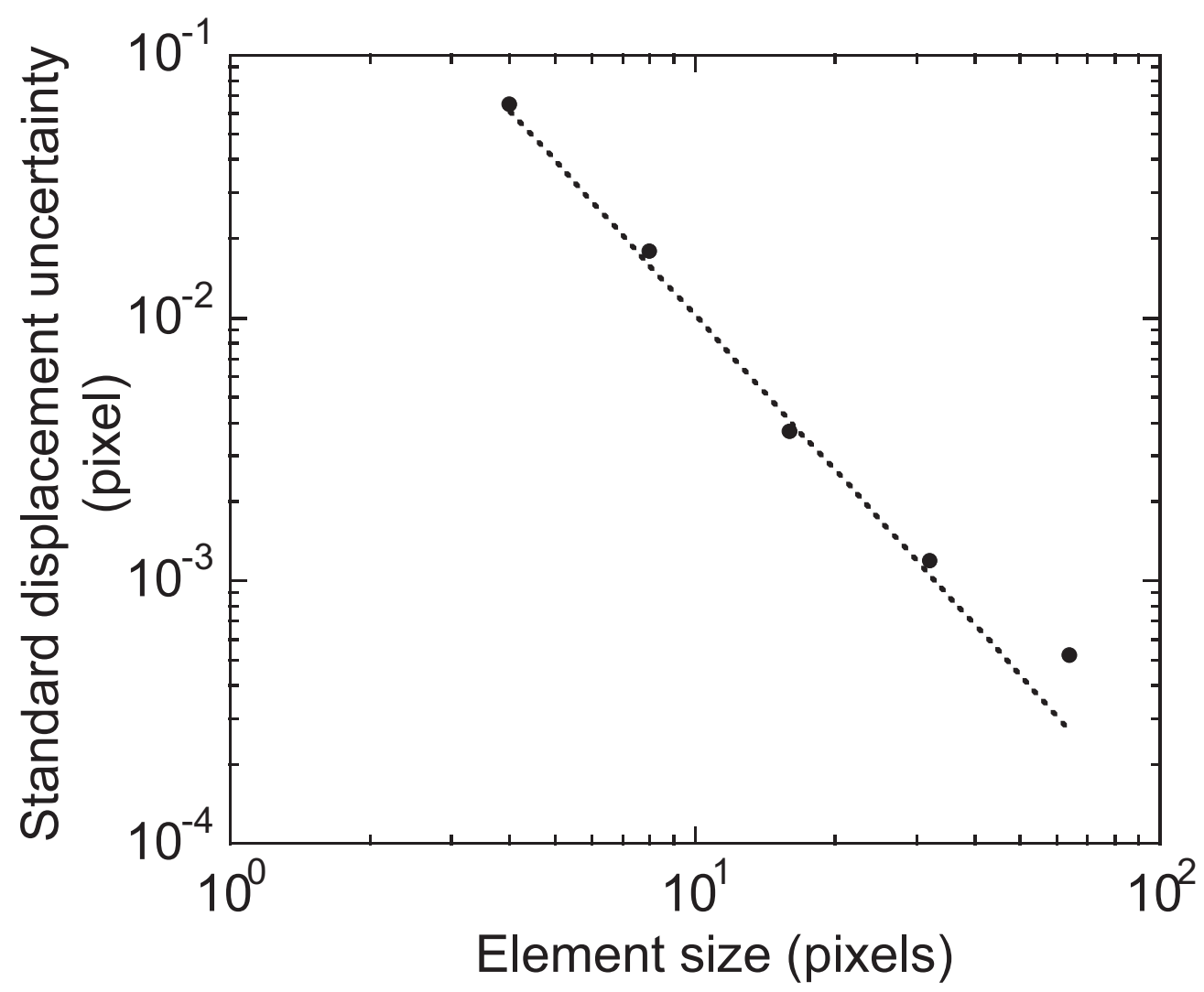

Fig. 3. Standard displacement uncertainty as a function of element size $\lambda$ using Q4 finite elements. The dashed line corresponds to a power law fit [Equation (21)] with an exponent close to 2 . 


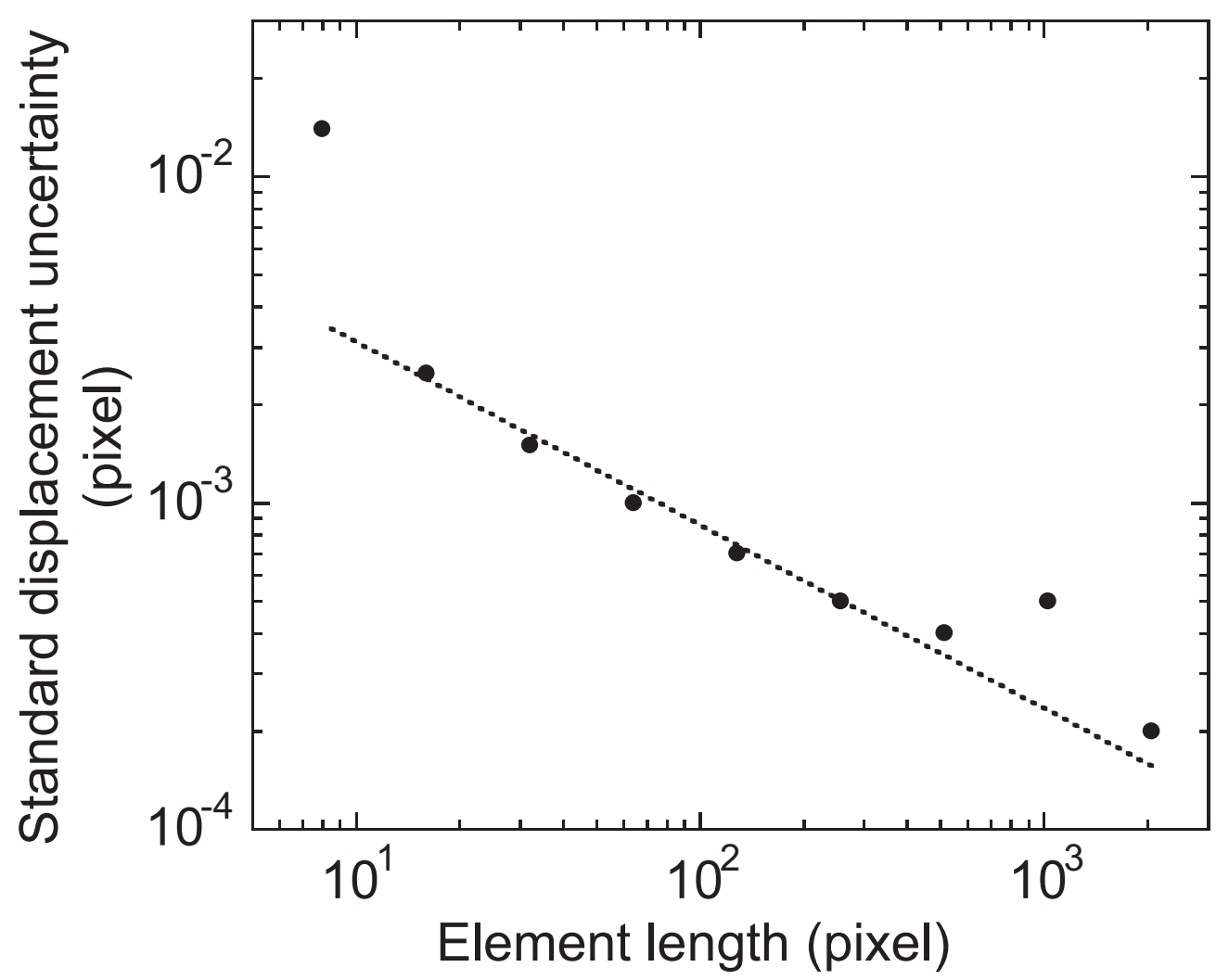

Fig. 4. Standard displacement uncertainty as a function of beam element length $\ell$. The dashed line corresponds to a power law fit with an exponent of the order of 0.6 . 

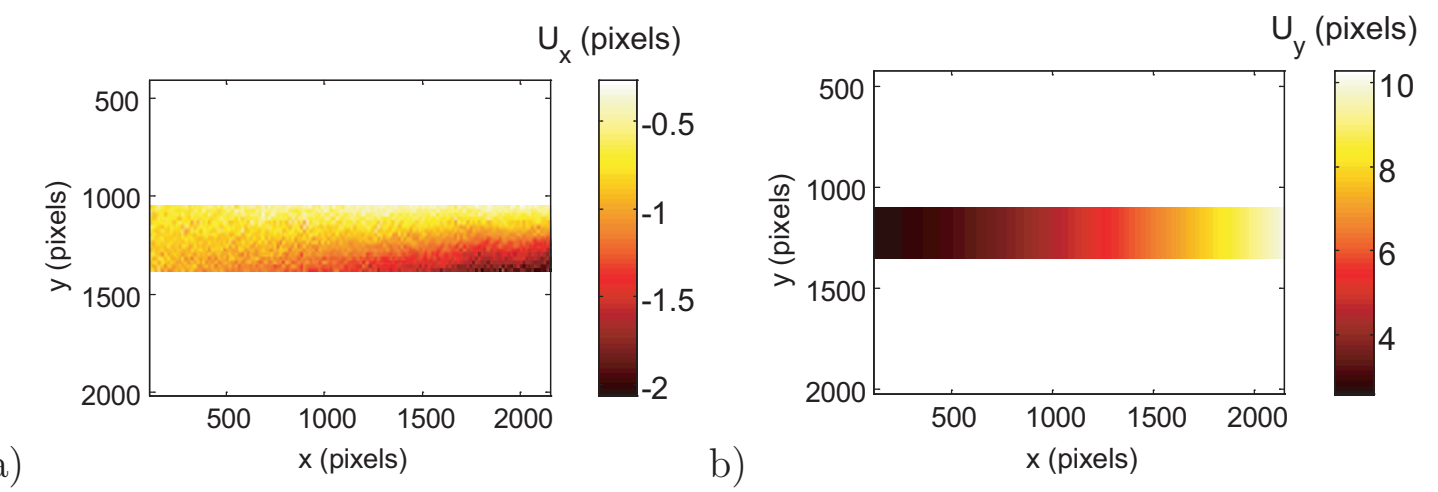

Fig. 5. Displacement maps in pixels $(1$ pixel $\equiv 0.35 \mathrm{~mm})$ along the longitudinal and transverse directions measured with a Q4-algorithm. Elements of size $\ell=16$ pixels were considered. 


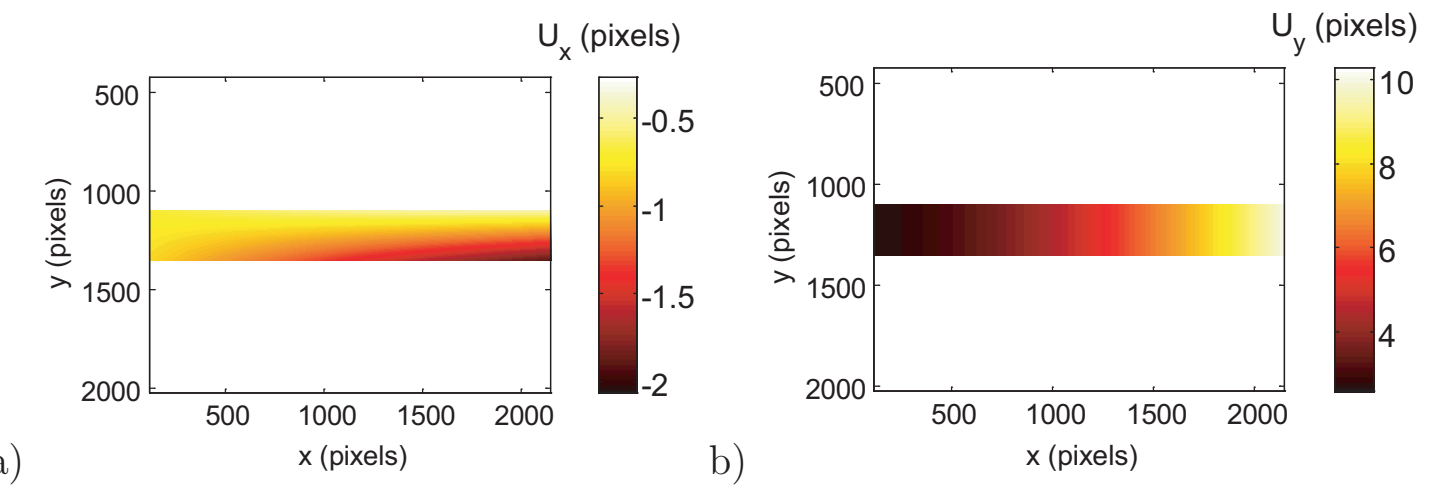

Fig. 6. Post-processed displacement maps using one beam element along the longitudinal and transverse directions. The same color scales as in Figure 5 are chosen. 


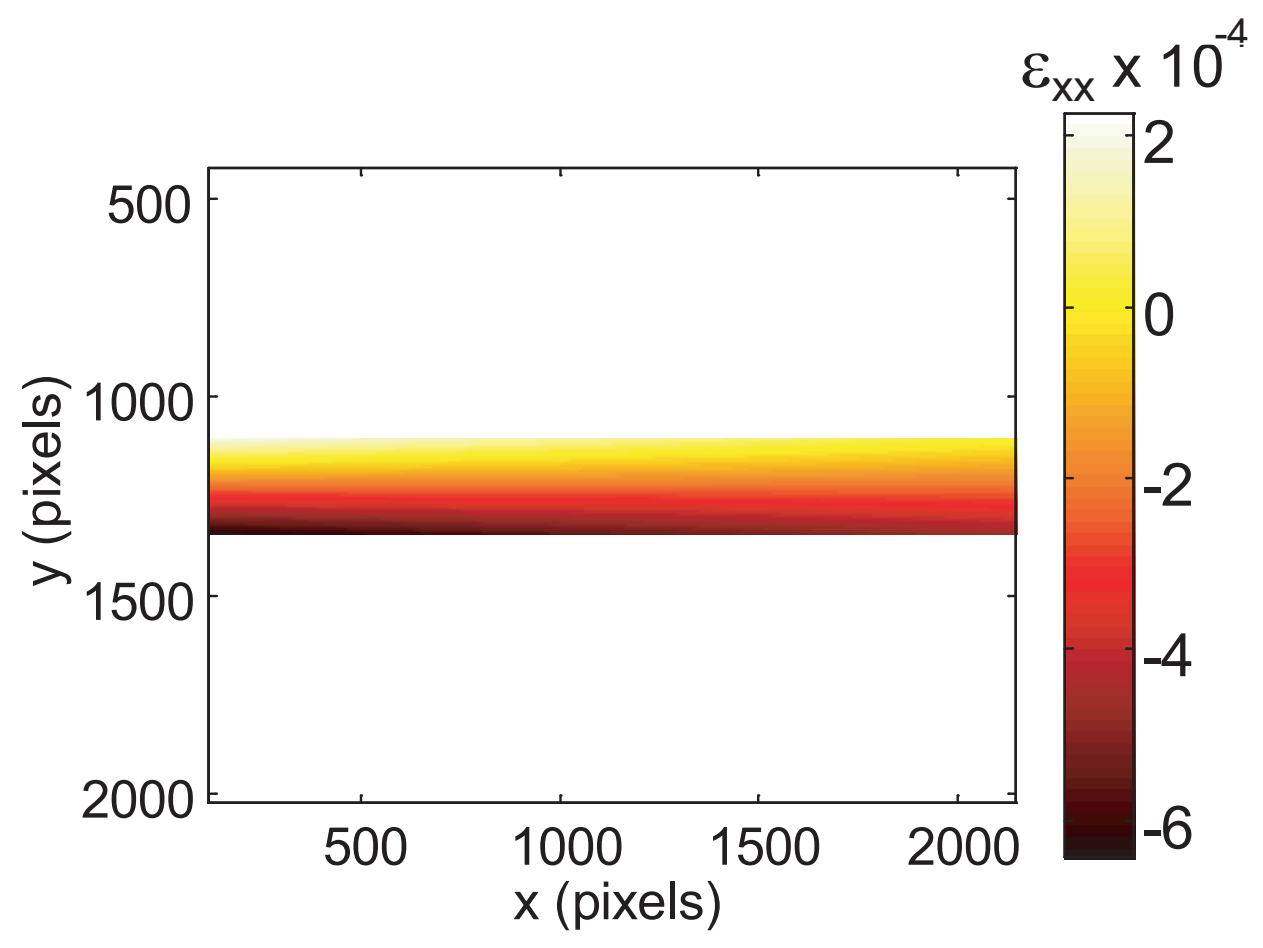

Fig. 7. Axial strain map obtained from post-processed displacements using one beam element (Figure 6) 


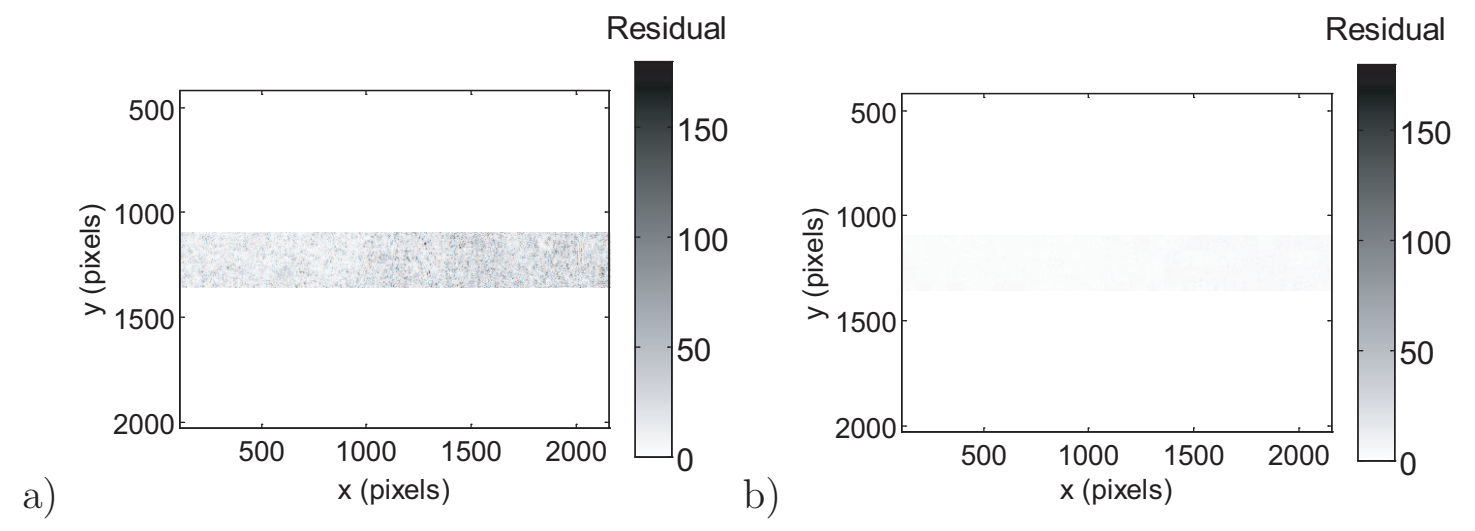

Fig. 8. Residual error map in gray levels prior to (a) and after (b) performing the correlation. One beam element of length $\ell=2048$ pixels is considered. 

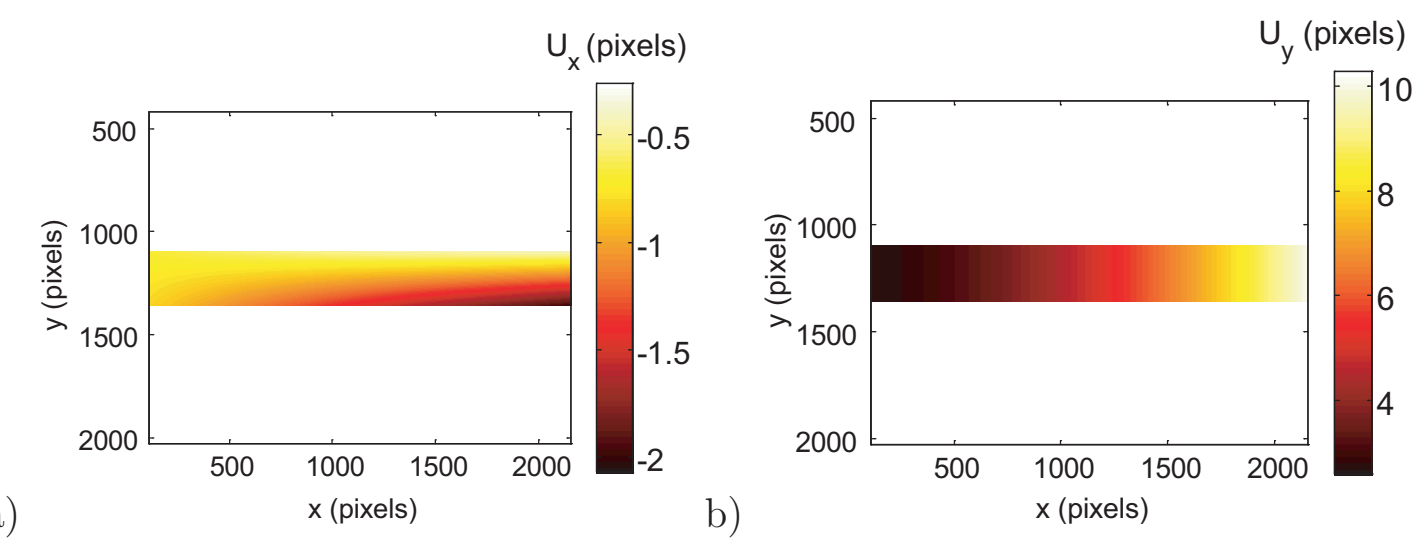

Fig. 9. Displacement maps along the longitudinal and transverse directions. One beam element of length $\ell=2048$ pixels is considered. The same color scales as in Figure 5 are chosen. 

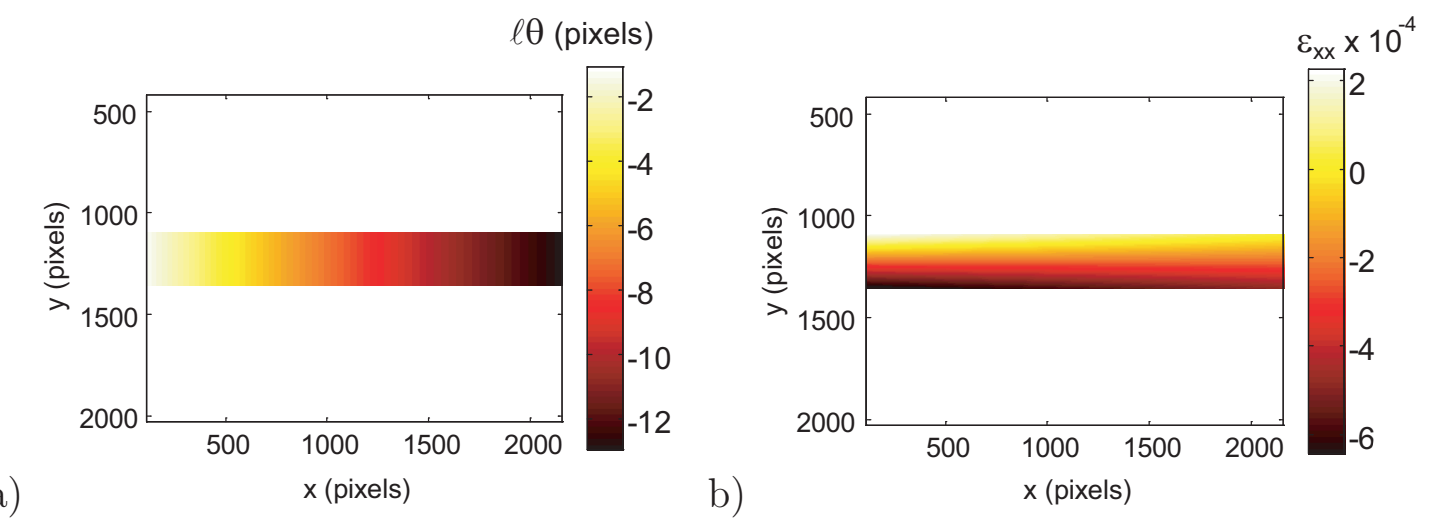

Fig. 10. Rotation (a) and axial strain (b) maps (the same color scale as in Figure 7 is chosen). One beam element of length $\ell=2048$ pixels is considered. 


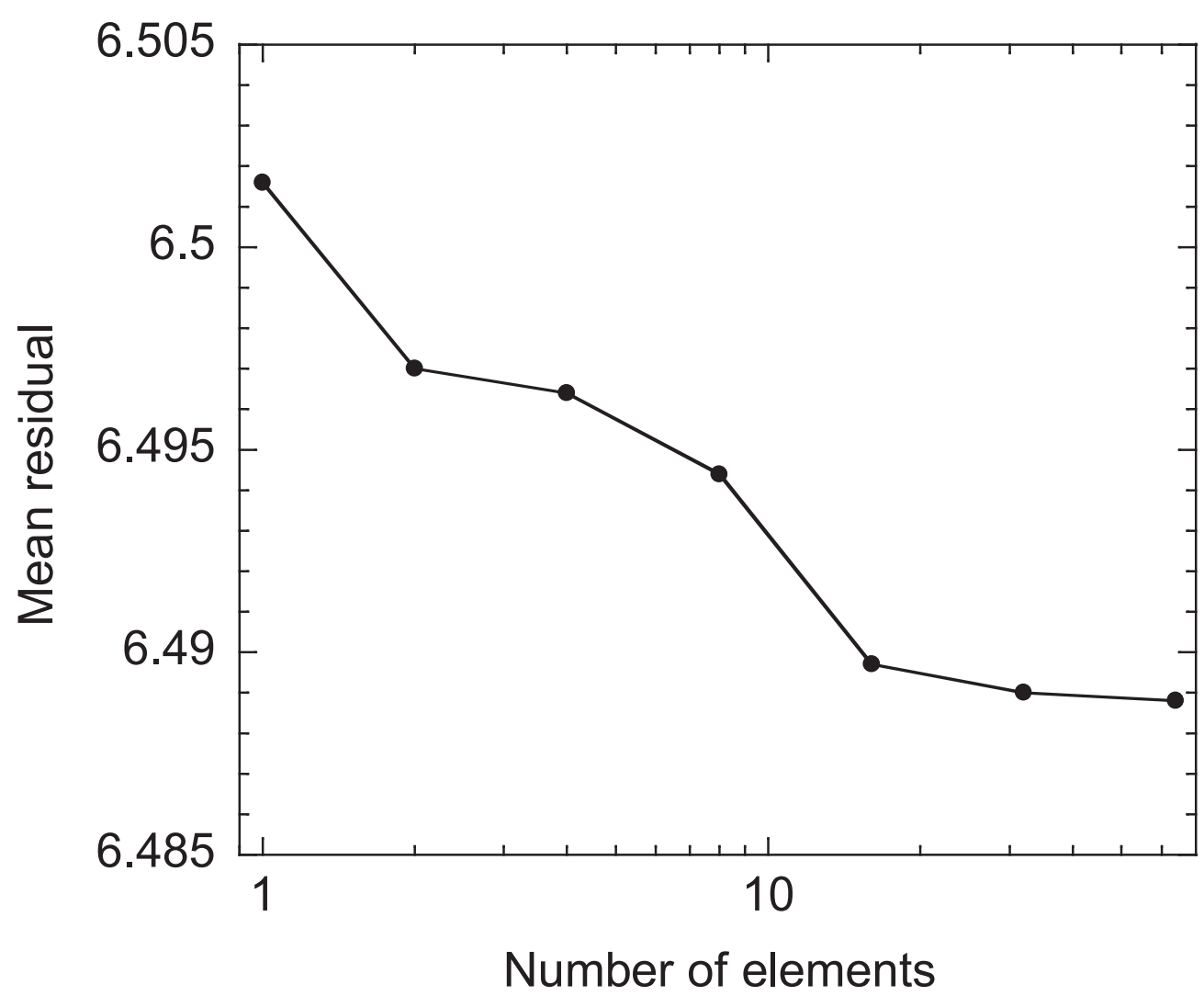

Fig. 11. Average residual (in gray levels) as a function of number of beam elements. The average initial residual (i.e., the picture difference) is equal to 28 gray levels. 


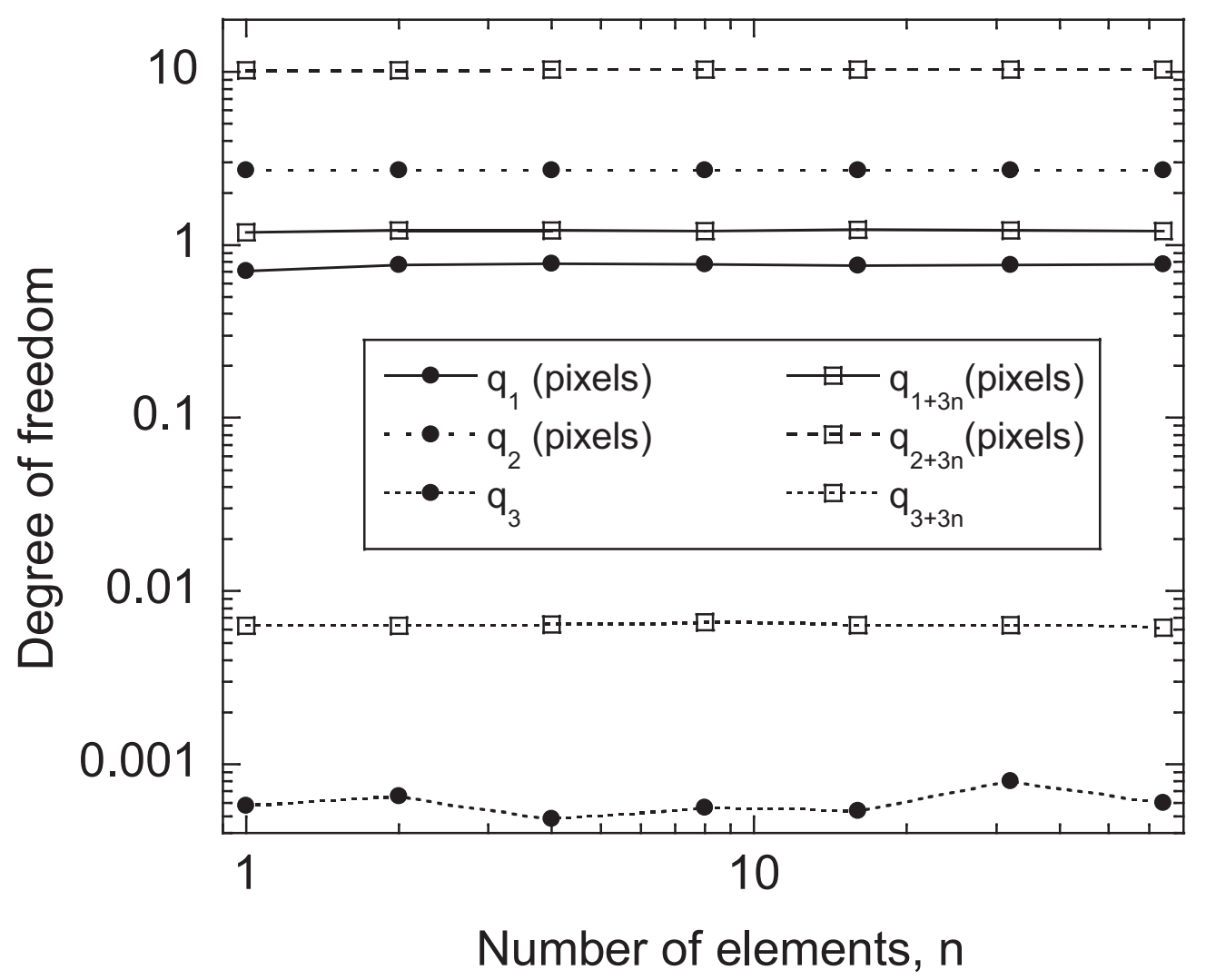

Fig. 12. Degrees of freedom as functions of number of beam elements. 


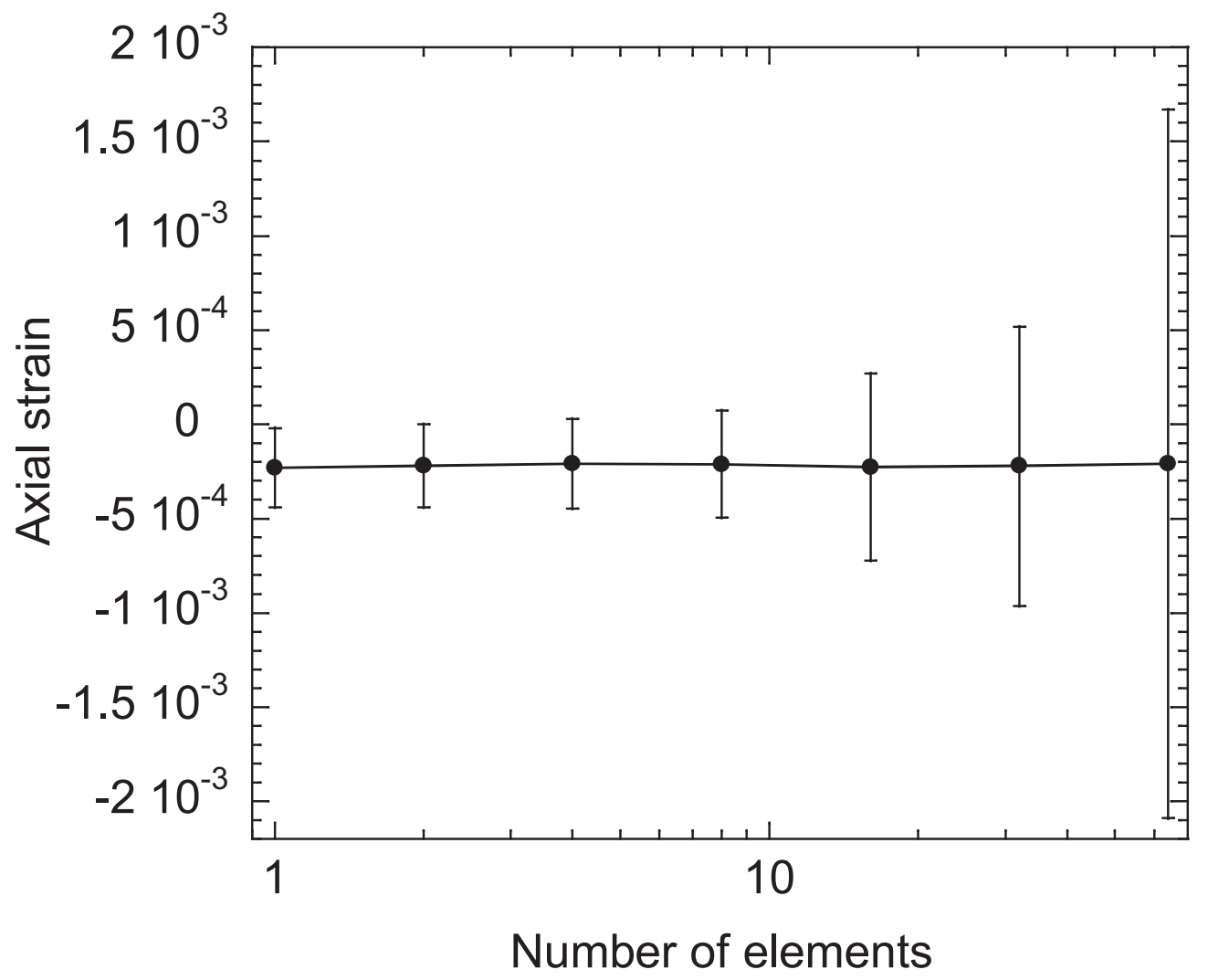

Fig. 13. Average axial strain as a function of number of beam elements. The error bars correspond to the root mean square values. 


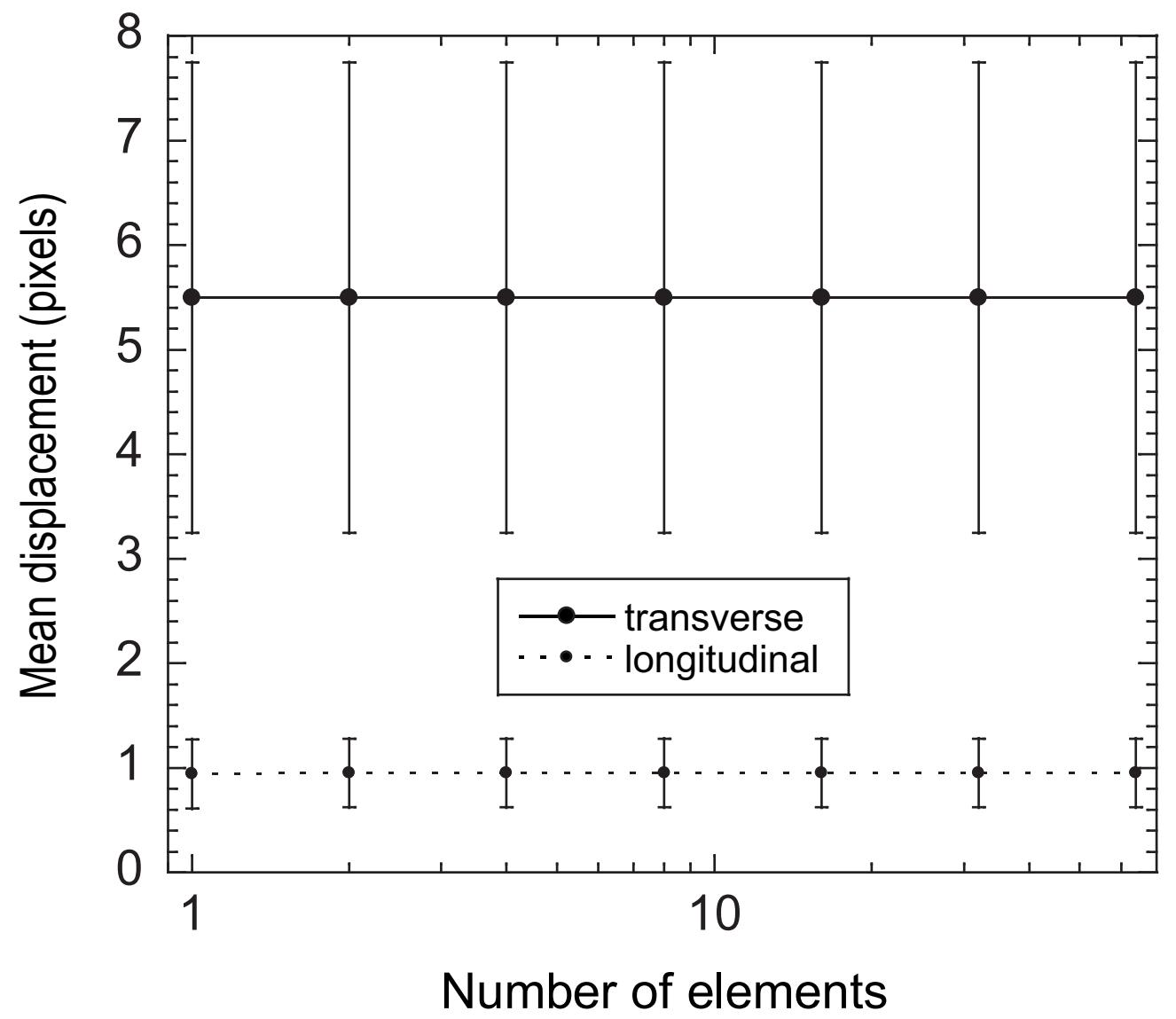

Fig. 14. Average displacements as functions of number of beam elements. The error bars correspond to the root mean square values. 

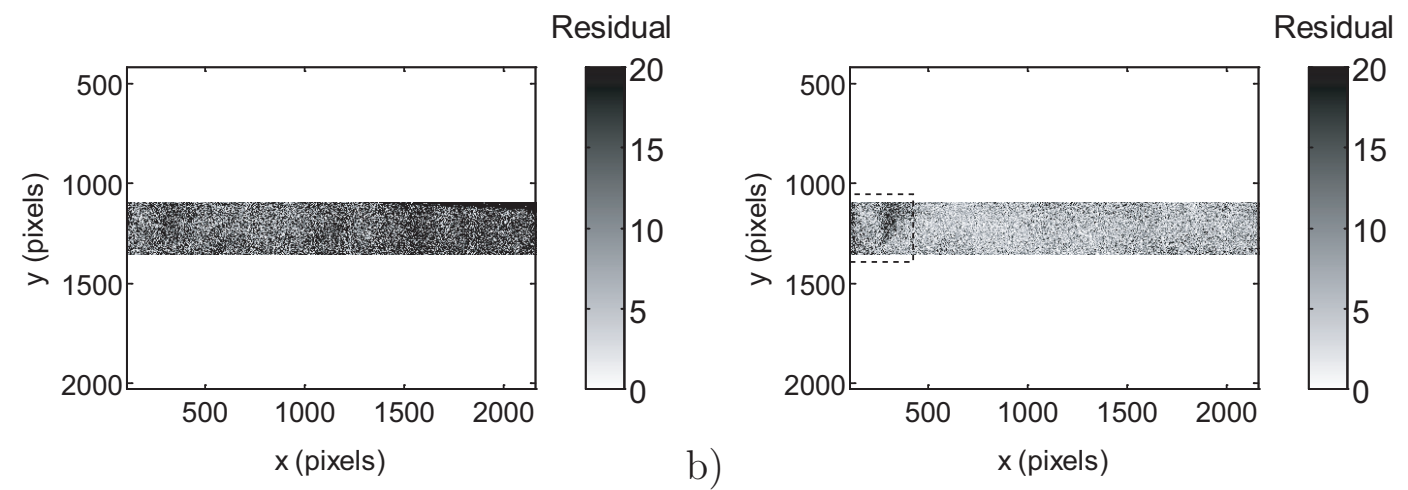

Fig. 15. Residual error maps prior to correlation (left) and after convergence (right) for the deformed picture shown in Figure 2. The dashed box shows the zone where the correlation result is not accurate. One beam element of length $\ell=2048$ pixels is considered. 


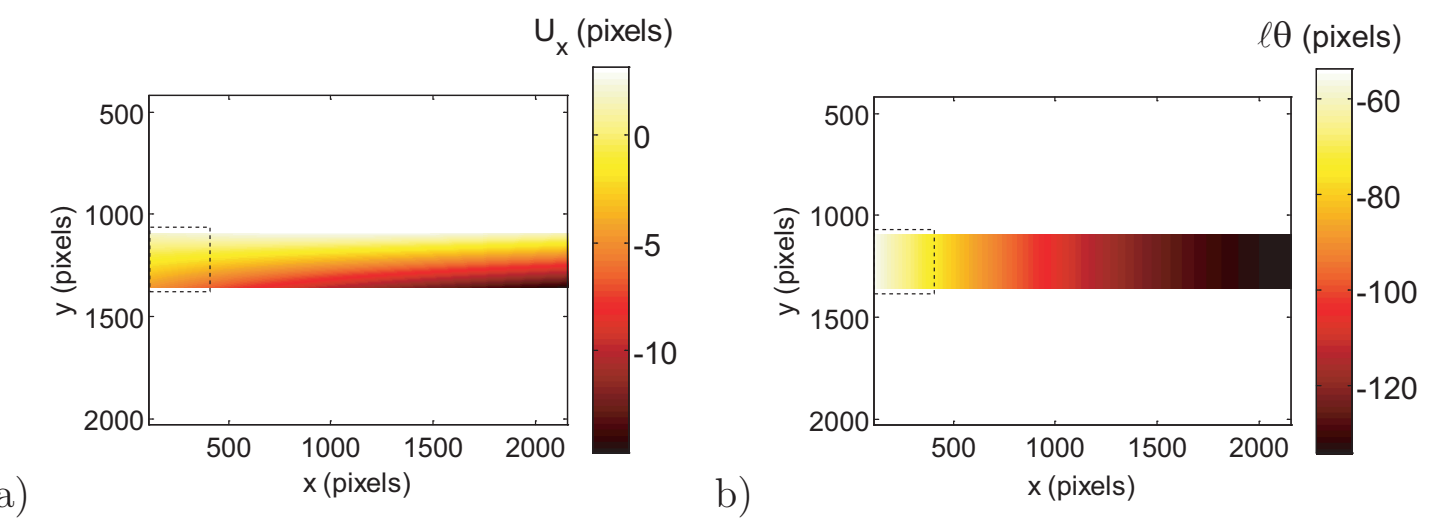

Fig. 16. Displacement map along the longitudinal direction and corresponding rotation map. The dashed box shows the zone where the measured kinematics is not trustworthy. One beam element of length $\ell=2048$ pixels is considered. 